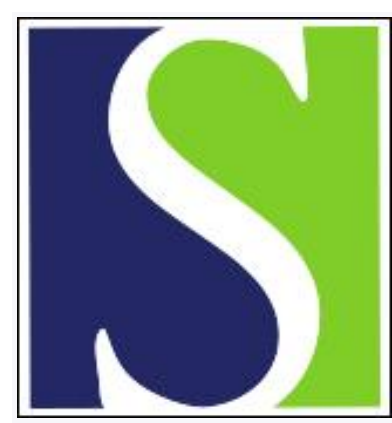

Scand J Work Environ Health 2000;26(1):62-66

https://doi.org/10.5271/sjweh.511

Issue date: Feb 2000

Analysis of urinary S-phenylmercapturic acid and trans, trans -muconic acid as exposure biomarkers of benzene in petrochemical and industrial areas of Korea

by Fang MZ, Shin MK, Park KW, Kim YS, Lee JW, Cho MH

Key terms: biomonitoring; environmental contamination

This article in PubMed: www.ncbi.nlm.nih.gov/pubmed/10744179

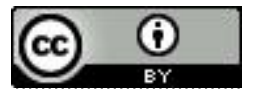




\title{
Analysis of urinary S-phenylmercapturic acid and trans,trans-muconic acid as exposure biomarkers of benzene in petrochemical and industrial areas of Korea
}

\author{
by Ming Zhu Fang, MSc, ${ }^{1}$ Min Ki Shin, BSc, ${ }^{1}$ Ki Wan Park, BSc, ${ }^{1}$ Yoon Shin Kim, PhD, ${ }^{2}$ Jee Woo Lee, \\ $P h D,{ }^{3}$ Myung Haing Cho, PhD'
}

Fang MZ, Shin MK, Park KW, Kim YS, Lee JW, Cho MH. Analysis of urinary S-phenylmercapturic acid and
trans, trans-muconic acid as exposure biomarkers of benzene in petrochemical and industrial areas of Korea.
Scand J Work Environ Health 2000;26(1):62-66.

\begin{abstract}
Objectives Recently, S-phenylmercapturic acid (S-PMA) and trans,trans-muconic acid $(t, t$-MA) in urine have been proposed as reliable biomarkers for monitoring occupational exposure to benzene. The aim of this study was to test the applicability of S-PMA and $t, t$-MA as exposure biomarkers and to monitor the occupational exposure level and the extent of environmental contamination from benzene in Korea.

Methods The urinary excretion of S-PMA and $t, t$-MA in rats after the intraperitoneal administration of benzene $(0.88 \sim 800 \mathrm{mg} / \mathrm{kg}$ body weight, 7 days) was examined. These biomarkers were also validated in human urine samples collected from elementary schoolchildren in several industrial areas including chemical manufacturing plants, oil refineries, and natural gas-producing installations in Korea. Urine was collected from elementary schoolchildren in a mountain village with no known occupational exposure to benzene and air pollution as the reference group.

Results In rats, there was a significant relationship between the benzene concentration and the excretion of the urinary S-PMA and $t, t$-MA as a function of concentration, and the excretion of benzene metabolites peaked on the first day after intraperitoneal administration. In human urine, higher levels of S-PMA and $t, t$-MA were detected more frequently in petrochemical industrial areas than in areas with no known occupational exposure to benzene. Conclusions These results show that the quantitative determination of S-PMA and $t, t$-MA in urine can be used as a reliable exposure biomarker for benzene, and they also suggest that extensive attention to benzene exposure is needed for maintaining the health of the population in Korea.
\end{abstract}

Key terms biomonitoring, environmental contamination.

Long-term exposure to high concentrations of benzene may lead to potentially adverse health effects, including bone marrow damage such as acute myeloid leukemia (1). For decades, as the steel and petrochemical industries have developed, serious problems with occupational exposure to benzene and air pollution in Korea have also been increasing proportionally. Because of more extensive attention to minimizing the exposure to benzene and to keeping the occupational exposure limit of airborne benzene as low as $1 \mathrm{ppm}$ (8-hour time-weighted average) in many countries, effective techniques for biological monitoring have been demanded for the detection and evaluation of such low concentrations of benzene. Benzene metabolites found in the urine are mainly the phenolic metabolites, muconic acid by the ring opening of the metabolite, and mercapturic acids formed by glutathione conjugation followed by hydrolysis. Since the main metabolites are phenol and its conjugates (figure 1) (2-3), urinary phenol has been used as a biomarker for occupational exposure to benzene for a long time. However, its use is limited to benzene concentrations over $5 \mathrm{ppm}\left(16 \mathrm{mg} / \mathrm{m}^{3}\right)$ due to its high and variable background because of the endogenous production of phenol and the high dietary and environmental

1 Laboratory of Toxicology, College of Veterinary Medicine, Seoul National University, Korea.

2 College of Medicine, Hanyang University, Korea.

3 College of Pharmacy, Seoul National University, Korea.

Reprint requests to: Myung-Haing Cho, Laboratory of Toxicology, College of Veterinary Medicine, Seoul National University, Suwon 441-744, Korea. [E-mail: mchotox@snu.ac.kr] 
exposure to phenol. In the past few years, trans,transmuconic acid ( $t, t$-MA) and S-phenylmercapturic acid (SPMA), 2 minor urinary metabolites of benzene, have attracted much attention as new biomarkers of benzene exposure at levels even below $1 \mathrm{ppm}$ (4).

In order to test the applicability of S-PMA and $t, t$ MA as exposure biomarkers in biomonitoring the occupational exposure levels and the extent of environmental pollution by benzene in Korea, the urinary excretion of S-PMA and $t, t$-MA was initially examined in rats, and then the methods were applied to 144 human urine samples to evaluate the levels of both metabolites.

\section{Material and methods}

\section{Materials}

Chemicals. Benzene ( $>99 \%$ ), S-phenylmercapturic acid (>98\%), and S-benzylmercapturic acid (>98\%) were purchased from Tokyo Kasei Organic Chemicals (Tokyo, Japan). The Hitrap Q column was purchased from Pharmacia (Hong Kong, China), and (trimethylsilyl)diazomethane and trans,trans-muconic acid (>99\%) were obtained from Aldrich Chemicals (Milwaukee, WI, USA). Other chemicals were of HPLC grade reagents or better grade and obtained from Sigma Chemicals (St Lous, MO, USA).

Samples. Urinary samples were collected from male Sprague Dawley rats after the intraperitoneal administration of various concentrations of benzene $(0.88 \sim 800 \mathrm{mg} /$ $\mathrm{kg}$ body weight) for 7 days. All rats weighing between 150 and 250 grams were obtained from Seoul National University Laboratory Animal Facility (Seoul, Korea).

Human urine samples (total 144) were collected from elementary schoolchildren (8-to-12-year-old healthy volunteers) in a petrochemical industrial area, a petrochemical industrial urban area, and a mountain village with no known occupational exposure to benzene and air pollution. In all studies, the urine samples were collected in polypropylene bottles and immediately transported to our laboratory to be stored at $-20^{\circ} \mathrm{C}$ until the analysis.

\section{Methods}

Determination of urinary creatinine. To adjust for the dilution of urine samples caused by differences in the fluid intake between persons, urinary creatinine was determined by the modified Jaffe method using a creatinine detection kit (Shinyang Chemical, Seoul, Korea).

Determination of urinary trans, trans-muconic acid. The measurement of $t, t$-MA was carried out according to the method of Boogaard \& van Sittert (5). To improve the recovery, urinary samples were brought to $\mathrm{pH} 8.5-9.5$ by the addition of $35 \%$ (weight/volume) aqueous sodium hydroxide solution and centrifuged for 10 minutes at 2000 revolutions/minute, then filtered through a $45-\mathrm{nm}$ filter before the sample clean-up using solid phase extraction. An aliquot of $1.00 \mathrm{ml}$ of alkaline urine was subsequently passed through some brands of the quaternary ammonium ion exchange resin (SAX column: Hitrap Q column). The column was washed with $3 \mathrm{ml}$ of $1 \%$ acetic acid (volume/volume), and the $t, t$-MA was eluted with $4 \mathrm{ml}$ of $10 \%$ (volume/volume) acetic acid into the calibrated tubes. The volume was then increased to $5.0 \mathrm{ml}$ with pure water. Aliquots $(100 \mu \mathrm{l})$ of this solution were analyzed by high-performance liquid chromatography (HPLC) with an ultraviolet (UV) detector $(\lambda=259 \mathrm{~nm})$ at $20^{\circ} \mathrm{C}$ using a symmetry $\mathrm{C} 18$ column $(4.6 \times 250 \mathrm{~mm})$ as the stationary phase and $20 \%$ (volume/volume) methanol in $1 \%$ (volume/volume) aqueous acetic acid as the mobile phase. The flow rate was set at $1 \mathrm{ml} / \mathrm{min}$. For calibration, a series of $t, t$-MA standards in fresh control urine samples were analyzed together with the urine samples. A more effective clean-up was alkalinization of the samples to lower the detection limit to $4.8 \mu \mathrm{g} / 1$. The coefficient of variation of the replicate analysis $(\mathrm{N}=6)$ was $1.6 \%$ and $1.85 \%$ at spiked concentrations of $8 \mathrm{mg} / \mathrm{l}$ and $15.6 \mu \mathrm{g} / 1$, respectively. The results of the urinary $t, t$ MA measurements have been expressed as micrograms per gram of creatinine.

Determination of S-phenylmercapturic acid. Urinary samples were acidified to $\mathrm{pH} 2$ with $6 \mathrm{M}$ hydrochloric acid. A $1.00-\mathrm{ml}$ aliquot of acidified urine containing $200 \mathrm{ng}$ of S-benzylmercapturic acid (S-BMA) as an internal standard was extracted with $4.0 \mathrm{ml}$ of ethyl acetate. After centrifugation, the ethyl acetate layer was evaporated to dryness under a gentle stream of nitrogen in a water bath at $45^{\circ} \mathrm{C}$, and the residue was redissolved in $0.5 \mathrm{ml}$ of tetrahydrofuran and then methylated by $0.2 \mathrm{ml}$ of methanol and $0.2 \mathrm{ml}$ of (trimethylsilyl) diazomethine $(2.0$ $\mathrm{M}$ in hexanes) through shaking for 2 hours at room

\section{benzene

$$
\downarrow
$$

muconaldehyde $\leftarrow$ benzene epoxide $\rightarrow \rightarrow \rightarrow \rightarrow$ glutathionyl-hydroxy-hexadiene

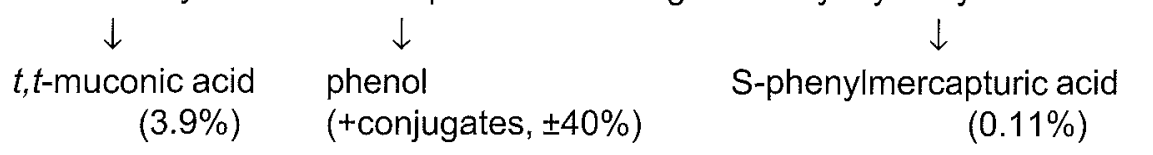

Figure 1. Some metabolic pathways of benzene and the urinary excretion of benzene metabolites. 
A

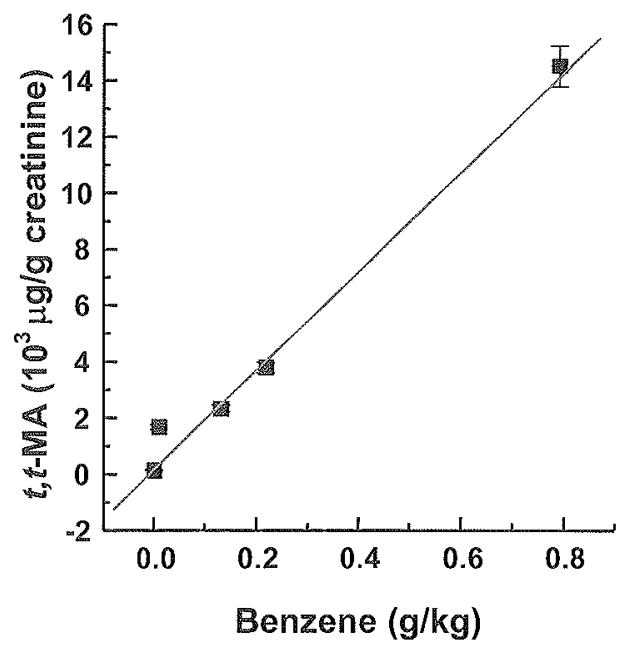

B

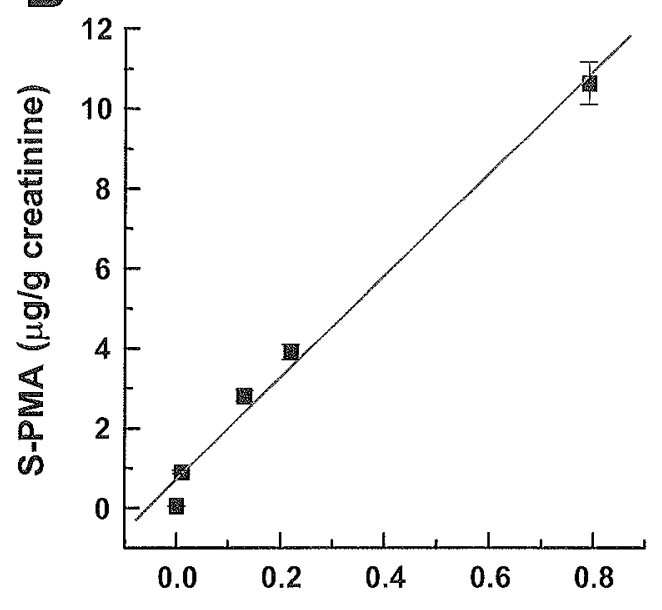

Benzene $(\mathrm{g} / \mathrm{kg})$

Figure 2. Relationship between the concentration of benzene administered intraperitoneally to rats and the concentration of trans, transmuconic acid $(t, t-\mathrm{MA})(\mathrm{A})$ and $\mathrm{S}$-phenylmercapturic acid (S-PMA)(B) in urinary samples collected in the first 24 hours after the exposure. The values represent means and their standard deviations $(N=4)$.

temperature. After methylation, the solution was evaporated to dryness under a gentle stream of nitrogen in a water bath at $45^{\circ} \mathrm{C}$ and redissolved in $1.0 \mathrm{ml}$ of dichloromethane. The samples were analyzed by gas chromatography mass spectrometry selected-ion recording (Hewlett-Packard HP 5971 Series Mass Selective Detector, interphased with a Hewlett-Packard HP 5890 series II, gas chromatograph, equipped with HP MS ChemStation system software). An HP-5 (cross-linked phenyl methyl silicone, $25 \mathrm{~m} \times 0.2 \mathrm{~mm}$ inner diameter $\times$ film thickness $0.33 \mu \mathrm{m}$ ) capillary column was used. The carrier gas was high-purity helium gas (flow of about 0.6 $\mathrm{ml} / \mathrm{min}$ ). The oven temperature program was $150^{\circ} \mathrm{C}$ for $1 \mathrm{~min}$, then $10^{\circ} \mathrm{C} / \mathrm{min}$ steps up to $280^{\circ} \mathrm{C}$, then constant at $280^{\circ} \mathrm{C}$ for 2 minutes. The temperatures of the injector, transfer line of MSD and ionization source were $250^{\circ} \mathrm{C}$,

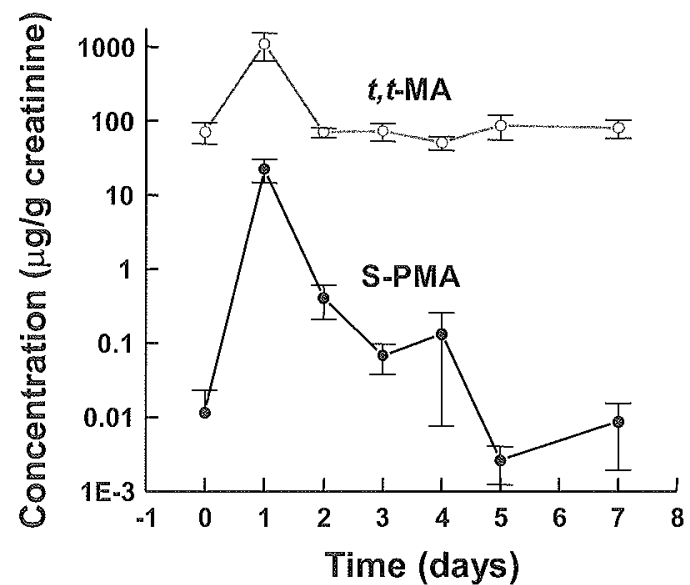

Figure 3. Urinary excretion of trans, trans-muconic acid $(t, t-\mathrm{MA})$ and S-phenylmercapturic acid (S-PMA) from rats after the intraperitoneal administration of benzene ( $0.3 \mathrm{~g} / \mathrm{kg}$ body weight) on 7 consecutive days. The values represent means and their standard deviations $(N=4)$.

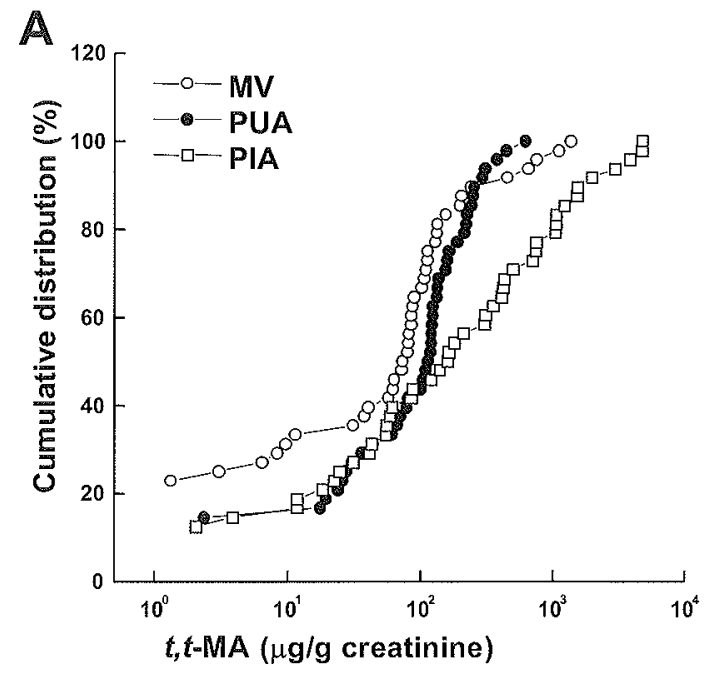

B

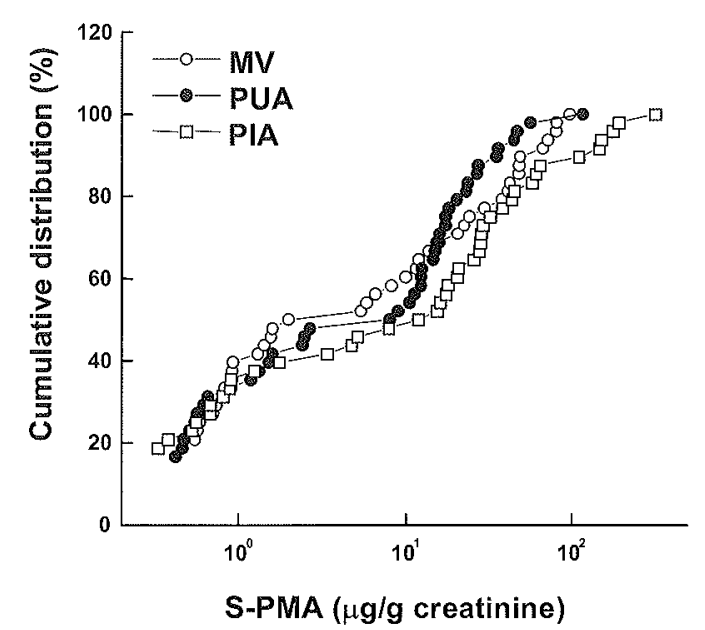

Figure 4. Cumulative frequency distributions of concentrations of trans,trans-muconic acid $(t, t-M A)(A)$ and S-phenylmercapturic acid (S-PMA) (B) ( $\mu \mathrm{g} / \mathrm{g}$ creatinine) in human urine in a mountain village (MV), a petrochemical urban area (PUA), and a petrochemical industrial area (PIA). 
$300^{\circ} \mathrm{C}$ and $200^{\circ} \mathrm{C}$, respectively. The solvent delay time was 3 minutes. The injection volume was $2 \mu \mathrm{l}$. For the quantification of S-PMA the ions at $m / z, 194$ (fragment of S-PMA methylester) and at $m / z 176$ (fragment of SBMA methylester) were monitored.

For calibration, a series of S-PMA standards in fresh control urine samples $(2-8000 \mu \mathrm{g} / \mathrm{l})$ were analyzed together with the urine samples. The ratio of the S-PMA methyl ester and the S-BMA methyl ester was plotted against the response ratio of $\mathrm{m} / z, 194$ and $\mathrm{m} / z$ 176. The recovery of added S-PMA was about $80 \%$ and the detection limit was $0.5 \mu \mathrm{g} / 1$. The results of the urinary $S$ PMA measurements have been expressed as micrograms per gram of creatinine.

\section{Results}

Relationship between exposure concentration, time and urinary excretion of trans, trans-muconic acid and $S$-pheny/mercapturic acid in rats

The relationships between the concentrations of benzene uptake, urinary $t, t$-MA, and S-PMA in samples collected in the first 24 hours after the intraperitoneal administration of benzene $(0.88-800 \mathrm{mg} / \mathrm{kg}$ body weight) are shown in figure 2 . For rats, there was a significant linear relationship between administration and the excretion of the urinary S-PMA and $t, t$-MA as a function of concentration (A: $r=0.903$, B: $r=0.905$ ).

Figure 3 depicts the concentrations of $t, t$-MA and SPMA in rats. The urinary excretion of the metabolites peaked on the first day after the intraperitoneal administration. It is clear, especially from the excretion profile on the first day, that the excretion of the 2 benzene metabolites follows roughly the same pattern, but, due to the shorter apparent half-time of $t, t$-MA, the $t, t$-MA concentration returns to the baseline value on the second day. It is also evident that the level of $t, t$-MA is $100-1000-$ fold higher than that of S-PMA.

Human urinary concentrations of trans, trans-muconic acid and S-phenylmercapturic acid in a petrochemical industrial area, a petrochemical urban area and a mountain village

The benzene metabolites $t, t$-MA and S-PMA were detected in 144 human urine samples collected from elementary schoolchildren in a petrochemical industrial area, a petrochemical urban area, and a mountain village with no exposure to benzene. The cumulative frequency distributions of the urinary concentrations of $t, t$-MA and S-PMA in the 3 different areas are shown in figure 4. Figure 4 shows that, in human urine, high concentrations of S-PMA and $t, t$-MA were detected more frequently in the petrochemical industrial areas than in the mountain village with no exposure to benzene, especially $t, t$-MA. But there were several children with higher excretion levels of S-PMA or $t, t$-MA in the mountain village.

Relationship between urinary trans, trans-muconic acid and $S$-pheny/mercapturic acid

In figure 5 , the $t, t$-MA concentrations were plotted against the S-PMA concentrations in the same urine sample, both after logarithmic transformation, for 37 rats (A) and 144 humans (B). Significant correlations between
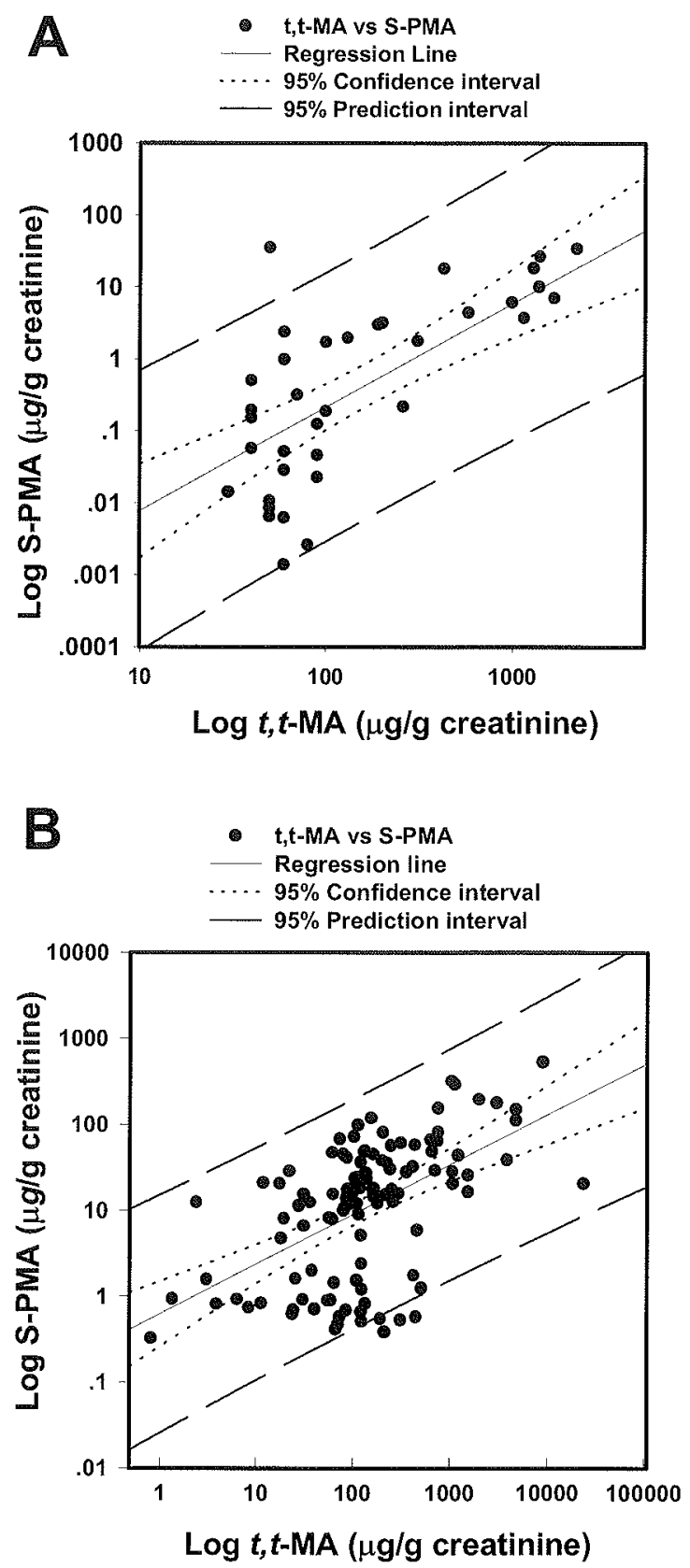

Figure 5. Relationship between the urinary concentration of trans, transmuconic acid ( $t, t-\mathrm{MA})$ and S-phenylmercapturic acid (S-PMA) in rats $(A)$ and humans $(B)$. 
$t, t$-MA and S-PMA were found $\left[\mathrm{r}^{2}=0.511(\mathrm{~A}), \mathrm{r}^{2}=0.294\right.$ (B)]. Nevertheless, some deviations were found. In some cases, relatively high concentrations of S-PMA (up to $5 \mu \mathrm{g} / \mathrm{g}$ creatinine) were detected in the urine (without a high concentration of $t, t-\mathrm{MA})$.

\section{Discussion}

Benzene occurs in the environment, primarily in the air. It is derived mainly from the emission from automobiles and the combustion processes in the petrochemical industry. However, residues are rarely found in water, food, and beverages. More than $99 \%$ of the total personal exposure takes place through air. The major cause of benzene exposure in smokers is smoking, approximately $80 \%$ of benzene exposure. But, in nonsmokers, the major source of benzene exposure comes from outdoor air, which makes up about $40 \%$ of the total exposure (6). Thus the values of the urinary metabolites of benzene in elementary schoolchildren can reflect the extent of environmental benzene exposure.

In the Woolsan industrial area in Korea, there are about 150 petrochemical manufacturing plants, $600 \mathrm{au}$ tomobile machine installation factories, and 50 nonferrous mineral plants. Results from the lung function tests of elementary schoolchildren from this area have confirmed the effect of air pollution on human health.

Furthermore, the data collected from this area revealed that benzene was one of the major constituents of contaminated air in these areas (unpublished observations). Recently, in a number of countries, the occupational exposure limit for airborne benzene has been lowered to $1 \mathrm{ppm}$ (8-hour time-weighted average). Weisel et al (7) reported that automobile mechanics were exposed to a mean concentration of $1 \mathrm{ppm}$, and workers at a chemical plant had benzene exposure with a geometric mean level of $0.1 \mathrm{ppm}$ for both smokers and nonsmokers. Therefore, it is very meaningful to verify the suitability of S-PMA and $t, t$-MA as exposure biomarkers which allow the detection of low concentrations of benzene and to apply their biomonitoring for the monitoring of occupational exposure and environmental pollution from benzene in Korea.

Even though the main benzene metabolites found in the urine are phenol and its conjugates, these substances are not suitable biomarkers because benzene exposure at levels below approximately 5 ppm cannot be detected when it is used as a marker, probably because of the high background levels caused by endogenous, dietary, and environmental sources of phenol. As a result, $t, t-\mathrm{MA}$ and S-PMA have been introduced as new biomarkers because of their ability to detect benzene exposure at levels below 1 ppm.
In our study, the relations between exposure to benzene and urinary $t, t$-MA $(r=0.903)$ and S-PMA $(r=0.905)$ in rats, as a function of dose, were linear. The urinary $S-$ PMA and $t, t$-MA concentrations in rats $(\mathrm{r}=0.71)$ and humans ( $r=0.54$ ) also correlated well.

These results support S-PMA and $t, t$-MA as reliable biomarkers for benzene exposure. These biomarkers were validated in human urine collected from elementary schoolchildren in several industrial areas in Korea. High levels of S-PMA and $t, t$-MA concentrations were generally and more frequenctly detected in the petrochemical industrial areas than in a mountain village with no exposure to benzene. Several children with a high excretion level of S-PMA or $t, t$-MA were found in areas with no occupational and environmental exposure. This result can be explained by indoor benzene sources such as automobile vapor emissions in attached garages or stored gasoline or kerosene in the garage or the basement and tobacco smoke exposure at home.

In conclusion, our study showed that S-PMA and $t, t$ MA in urine can be used as reliable exposure biomarkers to benzene exposure, and it aroused special attention to the national health in petrochemical and industrial areas of Korea.

\section{Acknowledgments}

This study was supported by the Ministry of Environment G-7 Project.

\section{References}

1. Snyder R, Kalf GF. A perspective on benzene leukemogenesis. Crit Rev Toxicol 1994;24:177-209.

2. Inoue O, Seiji K, Nakatsuka H, Watanabe T, Yin SN, Li GL, et al. Urinary t,t-muconic acid as an indicator of exposure to benzene. Br J Ind Med 1989;46:122-7.

3. Stommel P, Müller G, Stücker W, Verkoyen C, Schöbel S, Norpoth K. Determination of S-phenylmercapturic acid in the urine-an improvement on the biological monitoring of benzene exposure. Carcinogenesis 1989;10:279-82.

4. Boogaard PJ, Nico J, van Sittert NJ. Suitability of S-phenyl mercapturic acid and $t, t$-muconic acid as biomarkers for exposure to low concentration of benzene. Environ Health Perspect 1996;104 suppl 6:1151-7.

5. Boogaard PJ, van Sittert NJ. Biological monitoring of exposure to benzene: a comparison between S-phenylmercapturic acid, t,t-muconic acid, and phenol. Occup Environ Med 1995;52:611-20.

6. Wallace L. Environmental exposure to benzene: an update. Environ Health Perspect 1996;104 suppl 6:1129-36.

7. Weisel C, Yu R, Roy A, Georgopoulos P. Biomarkers of environmental benzene exposure. Environ Health Perspect 1996;104 suppl 6:1141-6.

Received for publication: 13 November 1998 